\title{
Association between Dyslipidemia and Diabetic Retinopathy in Type 2 Diabetic Patients
}

\author{
Elagamy $A^{1 *, \#, ~ A l ~ E n a z y ~ B R ~}{ }^{2, \#}$ and AL Zaaidi SE \\ Saudi Arabia and Mansoura Ophthalmic Center, Faculty of Medicine, Mansoura University, Egypt \\ ${ }^{2}$ Badriah Rakad Al Enazy, Optometry and Vision Sciences, Optometrist, Prince Sultan Military Medical City, Riyadh, Saudi Arabia \\ ${ }^{3}$ Sultan E. AL-Zaaidi, Consultant of Ophthalmology, Prince Sultan Military Medical City, Riyadh, Saudi Arabia \\ ${ }^{\#}$ Elagamy $A$ and Al Enazy BR have contributed equally
}

${ }^{1}$ Amira Elagamy, Assistant Professor of Ophthalmology, Department of Optometry and Vision Sciences, College of Applied Medical Sciences, King Saud University,

*Corresponding author: Amira Elagamy, Assistant Professor of Ophthalmology, Department of Optometry and Vision Sciences, College of Applied Medical Sciences, King Saud University, Saudi Arabia and Mansoura Ophthalmic Center, Faculty of Medicine, Mansoura University, Egypt, E-mail: aelagamy@ksu.edu.sa

Received: 15 Jul, 2018 | Accepted: 02 Aug, 2018 | Published: 09 Aug, 2018

Citation: Elagamy A, Al Enazy BR, AL Zaaidi SE (2018) Association between Dyslipidemia and Diabetic Retinopathy in Type 2 Diabetic Patients. J Ophthalmic Stud 1(1): dx.doi.org/10.16966/2639-152X.111

Copyright: (C) 2018 Elagamy A, et al. This is an open-access article distributed under the terms of the Creative Commons Attribution License, which permits unrestricted use, distribution, and reproduction in any medium, provided the original author and source are credited.

\section{Abstract}

Purpose: This study was conducted to assess the association between dyslipidaemia, blood pressure and diabetic retinopathy (DR) in type 2 diabetic patients. In addition, evaluation of the association between dyslipidemia and diabetic macular edema (DME) was performed.

Design: This study was a retrospective and cross-sectional design, observational, and quantitative study.

Subjects and Methods: This study included 800 patients with type 2 DM (386 males and 414 females). The age range was between 35-55 years. They were divided into two groups: 400 patients have DR (group 1), and 400 patients have not been previously diagnosed with DR (group 2). History and medical records of each diabetic patient were reviewed. Also, ophthalmological records of all subjects were reviewed including visual acuity and Intra Ocular Pressure (IOP) measurement using Air puff tonometer, macular thickness measured by Fourier-Domain Optical Coherence Tomography (OCT) and retinal imaging performed using Optomap $200^{\circ}$ Ultra-Widefield (UWF) Digital retinal scan. Measurements of blood pressure for all patients were reviewed. The last blood samples were reviewed for all subjects between 6 months to 1 year prior to our ophthalmic examination. Serum total cholesterol (TC), high density lipoprotein-cholesterol (HDL-C), low lipoprotein density-cholesterol (LDL-C), triglycerides (TG), plasma glucose and glycated hemoglobin (HbA1c) were taken. In addition, body mass index (BMI) and 10-year risk of Atherosclerotic Cardiovascular Disease (ASCVD) were calculated.

Results: There was a statistically significant relationship between DM duration and DR $(P<0.0001)$. Group 1 showed a statistically significant higher systolic and diastolic blood pressure than group $2(P<0.0001) \&(P=0.0289)$ respectively. Also, group 1 documented a statistically significant higher levels of TC, LDL-C and low levels of HDL-C $\left(0.0013^{*},<0.0001^{*}\right.$ and $\mathbf{0 . 0 0 2 7}$ ) respectively. But no statistically significant difference was detected between the 2 groups relating to TG $(P=0.5478)$. Besides, patients with $D M E$ had a statistically significant high $L D L-C$ and low HDL-C levels than patients without DME $(P<0.002)$ and $(P<0.003)$ respectively.

Conclusion: This study documented significant association between DR and dyslipidemia in type 2 diabetic patients. In addition, the study confirmed that patients with DME had significant high LDL-C level and low HDL-C level than patients without DME. Moreover, the current study demonstrated significant association between DR and hypertension. Therefore, efficient control of hyperglycemia, dyslipidemia and hypertension would be of great value in delaying the progression of DR in these patients.

Keywords: Diabetic retinopathy; Diabetic macular edema; Dyslipidemia; Hypertension

Abbreviations: Diabetes Mellitus (DM); High Serum Low-Density-Lipoprotein Cholesterol (LDL-C); Low Serum High-Density-Lipoprotein Cholesterol (HDL-C); Diabetic Retinopathy (DR); American Diabetes Association (ADA); Total Cholesterol (TC); Triglyceride (TG); Diabetic Macular Edema (DME); Intra Ocular Pressure (IOP); Ultra-Widefield (UWF); Optical Coherence Tomography (OCT); National Screening Committee (NSC); Glycated Haemoglobin (HbA1c); Non-Proliferative Diabetic Retinopathy (NPDR); Proliferative Diabetic Retinopathy (PDR); Atherosclerotic Cardiovascular Disease (ASCVD); Body Mass Index (BMI); Blood Pressure (BP); Statistical Package for Social Sciences (SPSS); United Kingdom Prospective Diabetes Study (UKPDS); Multi-Ethnic Study of Atherosclerosis (MESA); Cardiovascular Health Study (CHS); Early Treatment Diabetic Retinopathy Study (ETDRS); Wisconsin Epidemiologic Study of Diabetic Retinopathy (WESDR) 


\section{Background}

Diabetes Mellitus (DM) is a severe metabolic illness, with an increasing frequency worldwide [1]. In Kingdom of Saudi Arabia, the incidence of DM was evaluated as $23.7 \%$ with more significant predominance in males compared to females $(26.2 \%$ and $21.5 \%$ respectively) [2].

Metabolic syndrome is identified as an association of type 2 DM and other conditions such as hypertension, high serum low-densitylipoprotein cholesterol (LDL-C) concentration, and low serum highdensity-lipoprotein cholesterol (HDL-C) concentration [3]

Several studies [4,5] have demonstrated that chronic hyperglycemia, hypertension and hyperlipidemia contribute to the pathogenesis of Diabetic retinopathy (DR) which is one of the most common causes of visual impairment in people of working age, affecting both genders equally. The onset and deterioration of DR can be delayed by effective metabolic control. Elevated glucose levels cause retinal microangiopathy but the strict pathogenesis by which hyperglycemia leads to vascular damage seen in DR is ill defined. On the other hand, many biochemical pathways have been proposed to prove association between hyperglycemia and retinal microvascular abnormalities [6].

As specified by the American Diabetes Association (ADA) 2016 [7] hypercholesterolemia was demarcated as total cholesterol (TC) $>200$ $\mathrm{mg} / \mathrm{dl}$, high LDL-C when value $>100 \mathrm{mg} / \mathrm{dl}$, hypertriglyceridemia as triglycerides (TG) $>150 \mathrm{mg} / \mathrm{dl}$ and low HDL-C when value $<40 \mathrm{mg} /$ dl. Dyslipidemia was well-defined by the presence of one or more than one abnormal serum lipid concentrations. Prescription of Statin treatment should be of great value in these cases.

The aim of this study was to assess the association between dyslipidemia, blood pressure and DR in type 2 diabetic patients. In addition, evaluation of the association between dyslipidemia and diabetic macular edema (DME) was performed.

\section{Design}

This study was a retrospective and cross-sectional design, observational, and quantitative study.

\section{Subjects and Methods}

This study got the approval of Research Ethics Committee of College of Applied Medical Sciences, King Saud University and Research Ethics Committee in Prince Sultan Military Medical City, Riyadh, Saudi Arabia. All procedures in this study were performed in accordance with Helsinki declaration and its later amendments. All the participants signed comprehensive consent after explanation of the possible consequences of the study prior to investigations. The participants were consecutively enrolled from the Department of Ophthalmology, Diabetic Center, Prince Sultan Military Medical City, Riyadh, Saudi Arabia from January 2015 to April 2015. Inclusion criteria included patients with type $2 \mathrm{DM}$ aged 35 years. A total of 800 patients (386 males and 414 females) were enrolled in this study. The age range was between $35-55$ years.

They were divided into two groups:

Group 1: 400 patients with type 2 DM have been labelled as DR by an ophthalmologist (Retina Specialist).

Group 2: 400 patients with type $2 \mathrm{DM}$ had not been previously diagnosed with retinopathy, nephropathy or other diabetes complications.

Exclusion criteria included patients with retinal disorders like vein/ artery occlusion, retinitis pigmentosa, vitreo-retinal degeneration and dystrophies. In addition, all secondary causes of retinal neovascularization, history of uveitis, history of glaucoma, type $1 \mathrm{DM}$ patients, and patients $<35$ years old were excluded.

For each patient, history and medical records were reviewed to determine the DM duration. Also, ophthalmological records of all subjects were reviewed including visual acuity assessment using Snellen visual acuity chart, refraction using Auto-refractometer (Topcon) and Intra Ocular Pressure (IOP) measurement using Air puff tonometer, macular thickness measured by Fourier-Domain Optical Coherence Tomography (OCT) (RTVue-100 software version: 6.8; Optovue) and retinal imaging performed using Optomap $200^{\circ}$ Ultra-Widefield (UWF) Digital retinal scan (Daytona) - Optos. The grading systems for DR used in this study were the National Screening Committee (NSC) classification and the International Clinical Diabetic Retinopathy Disease Severity Scale approved by American Academy of Ophthalmology [8]. The last blood samples for all subjects were reviewed. Readings of serum TC, LDL-C, HDL-C, TG, plasma glucose and glycated haemoglobin (HbAlc) were taken. LDL-C was calculated by Friedewald's formula. In addition, body mass index (BMI) and 10year risk of Atherosclerotic Cardiovascular Disease (ASCVD) were calculated. Additionally, measurements of blood pressure for all patients were reviewed. Hypertension was defined according to the current guidelines as BP levels $\geq 140 / 90 \mathrm{mmHg}$ or the use of antihypertensive drugs [9].

\section{Statistical Analyses}

The data were analysed using the statistical package for social sciences (SPSS) software (version 21). Data were presented as means \pm standard deviation or percentages. Differences between the studied groups were examined using unpaired t-test or the Mann-Whitney U-test for parametric and non-parametric data, respectively, while a chi-square test was used for categorical data. 10-year risk of ASCVD was calculated using heart risk calculator [10]. Assessment of the relation between dyslipidemia and DR was performed using one-way analysis of variance (ANOVA) with Tukey-Kramer Multiple Comparisons Test. P-value $<0.05$ was considered statistically significant.

\section{Results}

Demographic and clinical laboratory characteristics of all participants in both groups are shown in (Table 1). Regarding the relation between DR and DM duration in this study, a high statistically significant difference was demonstrated between the 2 groups $(\mathrm{P}<0.0001)$ (Figure 1). The percentage of DR had slightly increased in women versus men $(56.5 \%$ vs $43.5 \%)$, but without statistically significant difference (Figure 2). There was a statistically significant difference between the two groups relating to smoking $(\mathrm{P}<0.0001)$. There were $70 \%$ of smokers presented with DR compared to $30 \%$ of smokers without DR (Figure 3). Group 1 showed a statistically significant higher systolic and diastolic blood pressure than group 2 $(\mathrm{P}<0.0001)$ and $(\mathrm{P}=0.0289)$ respectively (Table 1, Figure 4$)$.

Regarding assessment of dyslipidemia: Group 1 documented a statistically significant higher levels of TC, LDL-C and low levels of HDL-C $\left(0.0013^{*},<0.0001^{*}\right.$ and $\left.0.0027^{*}\right)$ respectively. But no statistically significant difference was detected between the 2 groups relating to TG $(\mathrm{P}=0.5478)$ (Table 2$)$. This study documented a statistically significant association between the severity of DR and dyslipidemia, especially TC $(\mathrm{P}<0.001)$ and LDL-C level $(\mathrm{P}<0.003)$ (Table 3$)$.

Evaluation of the type of DR and presence of DME in this study: $64.05 \%$ of non-proliferative diabetic retinopathy (NPDR) patients had DME and $67.22 \%$ of proliferative diabetic retinopathy (PDR) patients 
Table 1: Demographic and clinical laboratory characteristics of all participants in both groups.

\begin{tabular}{|c|c|c|c|}
\hline Variable & With DR & Without DR & P ( 2 tailed) Unpaired $t$ test \\
\hline Male/Female (\%) & $212(53 \%) / 188(47 \%)$ & $197(49.25 \%) / 203(50.75 \%)$ & \\
\hline Mean age & $47.67 \pm 6.43$ & $46.30 \pm 6.70$ & 0.205 \\
\hline Diabetic duration (year) & $21.69 \pm 8.68$ & $18.62 \pm 8.54$ & $<0.0001^{*}$ \\
\hline Smokers (\%) & $205(51.25 \%)$ & $120(30 \%)$ & $<0.0001^{*}$ \\
\hline $\mathrm{TC}(\mathrm{mmol} / \mathrm{L})$ & $5.34 \pm 1.06$ & $5.10 \pm 1.03$ & $<0.0001^{*}$ \\
\hline $\mathrm{TG}$ (mmol/L) & $3.26 \pm 1.91$ & $3.26 \pm 1.65$ & 0.2624 \\
\hline HDL-C (mmol/L) & $1.24 \pm 0.41$ & $1.33 \pm 0.44$ & $0.0027^{*}$ \\
\hline LDL-C (mmol/L) & $3.05 \pm 0.74$ & $2.79 \pm 0.78$ & $<0.0001^{*}$ \\
\hline Hypertension (\%) & $318(79.5 \%)$ & $210(52.5 \%)$ & $<0.0001^{*}$ \\
\hline Systolic blood pressure (mm Hg & $137.49 \pm 17.58$ & $131.98 \pm 21.18$ & $<0.0001^{*}$ \\
\hline $\begin{array}{l}\text { Diastolic } \\
\text { blood pressure }(\mathrm{mm} \mathrm{Hg})\end{array}$ & $74.35 \pm 13.39$ & $72.30 \pm 13.14$ & $0.0289 *$ \\
\hline BMI $(\mathrm{kg} / \mathrm{m} 2)$ & $27.24 \pm 5.55$ & $28.68 \pm 6.00$ & $0.0005^{*}$ \\
\hline HbA1c & $10.06 \pm 1.76$ & $9.52 \pm 3.79$ & $0.0104^{*}$ \\
\hline ASCVD risk factor (\%) & $56 \%$ & $32 \%$ & $<0.0001^{*}$ \\
\hline
\end{tabular}

*P value $<0.05\left(^{*}\right)$ was considered statistically significant.

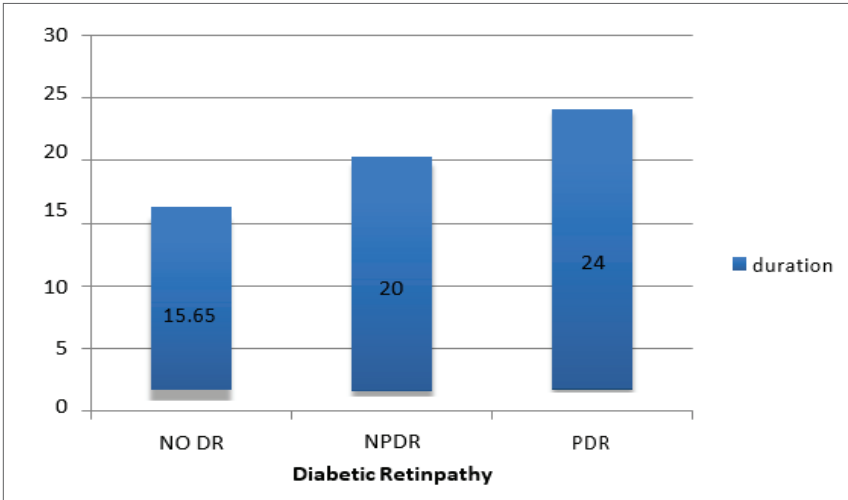

Figure 1: Association between diabetic retinopathy (DR) and diabetes mellitus (DM) duration.

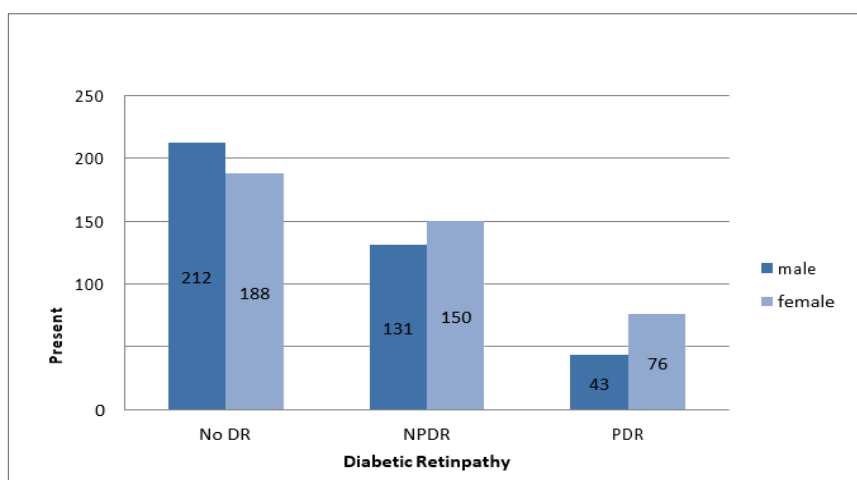

Figure 2: Association between diabetic retinopathy (DR) and sex.

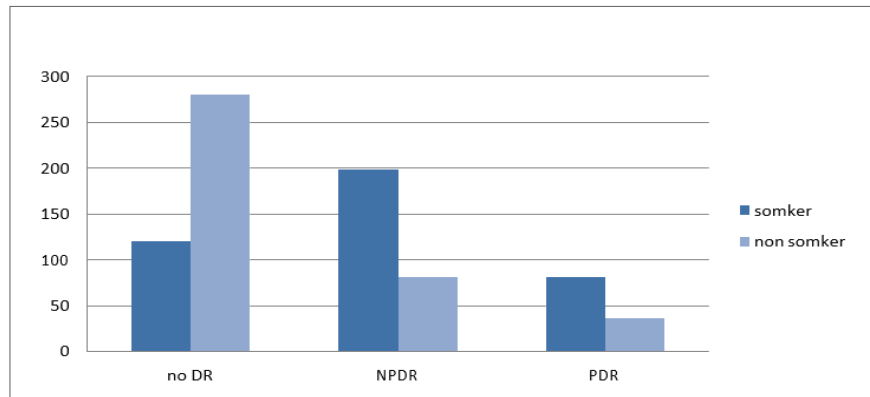

Figure 3: Association between diabetic retinopathy (DR) and smoking habit.

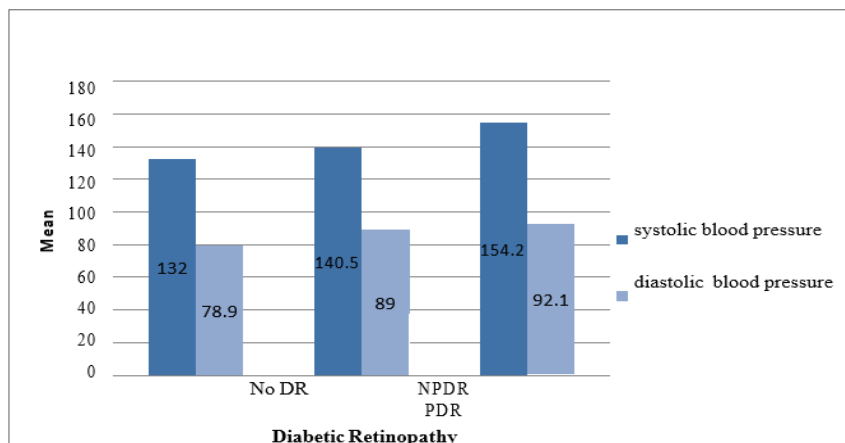

Figure 4: Association between diabetic retinopathy (DR) and blood pressure. 
Table 2: Association between dyslipidemia and diabetic retinopathy.

\begin{tabular}{|c|c|c|c|}
\hline \multirow{2}{*}{ Variable } & \multicolumn{2}{|c|}{ DR } & P value \\
\cline { 2 - 4 } & Absent & $5.25 \pm 1.05$ & $5.60 \pm 1.07$ \\
\hline TC $(\mathrm{mmol} / \mathrm{L})$ & $5.10 \pm 1.03$ & $3.86 \pm 1.91$ & $1.86 \pm 0.82$ \\
\hline TG (mmol/L) & $3.26 \pm 1.65$ & $1.24 \pm 0.44$ & $1.20 \pm 0.44$ \\
\hline HDL-C (mmol/L) & $1.33 \pm 0.44$ & $2.99 \pm 0.74$ & $3.14 \pm 0.72$ \\
\hline LDL-C (mmol/L) & $2.79 \pm 0.78$ & $39 \%$ & 0.5478 \\
\hline ASCDV risk factor (\%) & $32 \%$ & $<8 \%$ & $0.0016^{*}$ \\
\hline
\end{tabular}

*P value $<0.05\left({ }^{*}\right)$ was considered statistically significant.

Table 3: Association between dyslipidemia and severity of diabetic retinopathy.

\begin{tabular}{|l|c|c|c|c|}
\hline \multicolumn{1}{|c|}{ Grading of DR } & Mild NPDR & Moderate NPDR & Severe NPDR & PDR value \\
\hline TC(mmol/L) & $5.21 \pm 1.09$ & $5.29 \pm 1.10$ & $5.41 \pm 1.03$ & $5.60 \pm 1.07$ \\
\hline LDL-C level (mmol/L) & $2.79 \pm 0.78$ & $2.81 \pm 0.89$ & $2.87 \pm 0.58$ & $3.14 \pm 0.72$ \\
\hline HDL-C level (mmol/L) & $1.26 \pm 0.43$ & $1.23 \pm 0.32$ & $1.21 \pm 0.82$ & $1.20 \pm 0.44$ \\
\hline TG level (mmol/L) & $1.75 \pm 1.00$ & $1.82 \pm 0.63$ & $1.83 \pm 0.76$ & $1.86 \pm 0.82$ \\
\hline
\end{tabular}

$P$ value $<0.05\left(^{*}\right)$ was considered statistically significant.

Table 4: Number of patients according to the type of diabetic retinopathy and presence of diabetic macular edema (DME).

\begin{tabular}{|l|c|c|c|}
\hline \multicolumn{1}{|c|}{ Type of DR } & DME+ & DME- & Total \\
\hline NPDR & $180(64.05 \%)$ & 101 & 281 \\
\hline PDR & $80(67.22 \%)$ & 39 & 119 \\
\hline
\end{tabular}

had DME (Table 4). Table 5 shows number of patients according to the severity of DR and presence of DME. Patients with DME had a statistically significant high LDL-C and low HDL-C levels than patients without DME $(\mathrm{P}<0.002)$ and $(\mathrm{P}<0.003)$ respectively. But, no statistically significant differences were detected between the 2 groups concerning TG $(\mathrm{P}=0.180)$ and TC $(\mathrm{P}=0.151)$ (Table 6).

Regarding evaluation of 10-year risk of ASCVD: Group 1 showed a statistically significant higher ASCVD risk factor than group 2 (56\%) and $(32 \%)$ respectively $(\mathrm{P}<0.0001)$ (Table 2$)$. Figure 5 demonstrated ASCVD risk factor in patients without DR (32\%), with NPDR (39\%), and with PDR (48\%). There was a statistical significant difference between them $(\mathrm{P}<0.0318)$.

\section{Discussion}

DR is demonstrated in more than $60 \%$ of type $2 \mathrm{DM}$ patients during the first 20 years of the disease [11]. Many published articles found that poor glycemic control is markedly associated with DR [12]. However, some diabetic patients with modest hyperglycaemia show progressive DR, while others with highest hyperglycaemia over many years rarely demonstrate significant progression [13]. This may denote that DR pathogenesis may be attributed to other factors. There is a common link between DM and dyslipidemia. DR incidence depends on many factors such as the type and severity of diabetes, glycemic control, nutritional status, age and others [14]. On the other hand, the relation between pathogenesis of DR and dyslipidemia remains unclear. It is supposed that dyslipidemia is a risk factor for its development. DME is the most common cause of visual loss in diabetic patients and can occur at any stage of DR. Also, the link between pathogenesis of DME and dyslipidemia is still vague.
This study evaluated the association between DR, dyslipidemia, DME, and blood pressure in subjects with type $2 \mathrm{DM}$. The results showed that patients with DR had significantly higher values of TC and LDL-C and lower HDL-C levels compared to patients without DR. Although TG was higher in patients with DR, there was no statistically significant difference between the 2 groups. Also, our findings demonstrated presence of association between dyslipidemia and the presence and severity of DR. Our results matched with $\mathrm{Hu}$ et al. [15] study that reported significant association between low apolipoprotein A1/apolipoprotein B ratio in serum and PDR in type 2 diabetic patients of over 15 years' duration. In addition, Gadi and Samaha et al. [16] study demonstrated a strong prevalence of lipid abnormalities in patients with DR. Moreover, our findings agreed with Timothy et al. [17] study that documented positive association between the severity of retinopathy and TG level and negative association with HDL-C. Additionally, our findings matched with Ganeswaran et al. [18] study that showed significant association between DR and high levels of TC and LDL-C. On the other hand, the United Kingdom Prospective Diabetes Study (UKPDS) results documented that higher HDL-C level was associated with more severe DR. But, the reason of this association was not identified. However, the severity of DR was not related to TG level and LDL-C level [19]. Also, the Multi-Ethnic Study of Atherosclerosis (MESA) [20] evaluated the risk factors for DR in a multi-ethnic US population of whites, blacks, Hispanics, and Chinese and found no associations of plasma lipids and either retinopathy or macular edema in 778 individuals aged from 45 to 85 years with DM.

Our study showed a statistically significant difference between the 2 groups relating to DM duration. Regarding the sex, no statistically significant difference between the 2 groups was detected. In addition, we found a statistically significant difference between the 2 groups as regards to smoking. Additionally, level of systolic and diastolic blood pressure between the 2 groups showed a statistically significant difference. Nearly all participants in our study were overweight or obese with $\mathrm{BMI} \geq 25 \mathrm{Kg} / \mathrm{m}^{2}$.

Our findings concerning duration, sex, smoking, and blood pressure agreed with several studies such as, Al Aldebasi et al. [21] and El Mofty et al. [22] studies that documented a statistically significant relationship between DR and dyslipidemia and all these variables. 
Table 5: Number of patients according to the severity of diabetic retinopathy (DR) and presence of diabetic macular edema (DME).

\begin{tabular}{|c|c|c|c|c|c|c|c|c|c|c|}
\hline Grade of DR & 0 & 1 & 1+DME & 2 & 2+DME & 3 & 3+DME & 4 & 4+DME & Total \\
\hline Number of patients & 400 & 33 & 63 & 21 & 42 & 47 & 75 & 39 & 80 & 800 \\
\hline Incidence \% & 50 & 4.12 & 7.87 & 2.9 & 5.25 & 5.87 & 9 & 4.87 & 10 & 100 \\
\hline
\end{tabular}

Table 6: Assessment of dyslipidemia in diabetic retinopathy patients with \& without diabetic macular edema.

\begin{tabular}{|l|c|c|c|}
\hline & DR with DME & DR without DME & P value \\
\hline $\begin{array}{l}\text { TC } \\
\text { level (mmol/L) }\end{array}$ & $5.21 \pm 1.09$ & $5.29 \pm 1.10$ & 0.151 \\
\hline LDL-C level (mmol/L) & $2.82 \pm 0.78$ & $2.71 \pm 0.89$ & $<0.003^{*}$ \\
\hline HDL-C level (mmol/L) & $1.22 \pm 0.43$ & $1.63 \pm 0.32$ & $<0.002^{*}$ \\
\hline TG level (mmol/L) & $1.75 \pm 1.00$ & $1.82 \pm 0.63$ & 0.18 \\
\hline
\end{tabular}

*P value $<0.05\left({ }^{*}\right)$ was considered statistically significant.

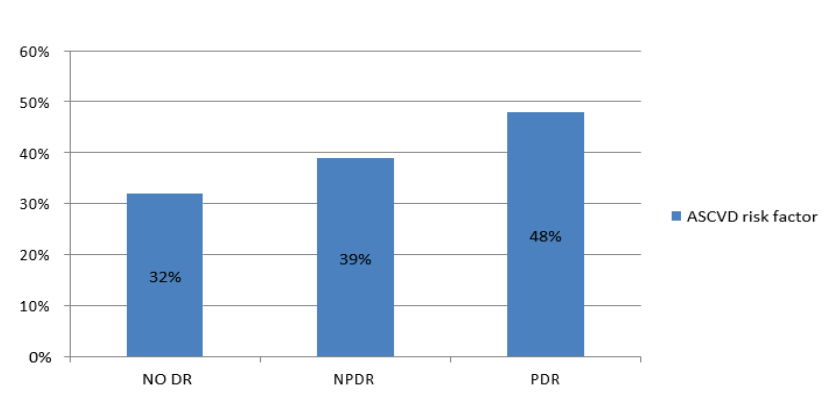

Figure 5: Atherosclerotic Cardiovascular Disease (ASCVD) risk factor in patients without diabetic retinopathy (DR) versus non-proliferative diabetic retinopathy (NPDR) patients and proliferative diabetic retinopathy $(P D R)$ patients.

Furthermore, the Hoorn Study [23] demonstrated positive association of DR with systemic hypertension, BMI, serum cholesterol and TG level. In addition, systemic hypertension and plasma TC and LDL-C levels found correlations with retinal hard exudates. Dyslipidemia in diabetics may be explained by specific abnormalities in lipoprotein metabolism and/or abnormalities in insulin action [23].

Concerning assessment of ASCVD risk factor, the current study showed a statistically significant difference between the 2 groups $(56 \%)$ and $(32 \%)$ respectively $(\mathrm{P}<0.0001)$ This finding matched with the Cardiovascular Health Study (CHS) which confirmed positive association of DR with higher average systolic blood pressure, higher TC and LDL-C levels, and the presence of cardiovascular disease [24].

Regarding relationship between dyslipidemia and DME in the current study, patients with DME have high LDL-C level and low HDL-C level than patients without DME. However, no statistically significant differences regarding TC and TG between the two groups were reported. These results agreed with the Hoorn [23] and Suwal et al. [25] studies that confirmed positive association between retinal hard exudates and high serum LDL-C level. Also, the Early Treatment Diabetic Retinopathy Study (ETDRS) [26] documented correlation between elevated TG and LDL-C and high risk of exudative maculopathy. Additionally, Wisconsin Epidemiologic Study of Diabetic Retinopathy (WESDR) XIII [27] demonstrated a significant association between increasing severity of DR and of retinal hard exudates and high TC in insulin-dependent diabetics. Likewise, Golubovic study [28] found significantly high levels of TG, TC, and cholesterol ester in type $2 \mathrm{DM}$ patients with DME compared to those without DME. But, there were no statistically significant differences of HDL-C and LDL-C levels between the 2 groups although higher values were detected in patients with DME. Moreover, Sachdev and Sahni study [29] in North India documented significant correlation between retinal hard exudates and systolic blood pressure, TC, LDL-C, and TG levels.

The differences between all the studies which might affect the results may be caused by many factors such as, sex, age, duration, race, and sample size of included subjects in each study.

\section{Conclusion}

This study documented significant association between DR and dyslipidemia in type 2 diabetic patients. In addition, the study confirmed that patients with DME had significant high LDL-C level and low HDL-C level than patients without DME. Moreover, the current study demonstrated significant association between DR and hypertension. Therefore, efficient control of hyperglycemia, dyslipidemia and hypertension would be of great value in delaying the progression of DR in these patients.

\section{Declarations}

Ethics approval and consent to participate: The study got the approval of Research Ethics Committee of College of Applied Medical Sciences, King Saud University and Research Ethics Committee in Prince Sultan Military Medical City, Riyadh, Saudi Arabia. All procedures performed in this study were in accordance with Helsinki declaration and its later amendments. All the participants signed comprehensive consent after explanation of the possible consequences of the study prior to investigations.

\section{Consent to publish: not applicable}

Availability of data and materials: not applicable

Competing interests: All authors certify that they have no affiliations with or involvement in any organization or entity with any financial interest (such as honoraria; educational grants; participation in speakers> bureaus; membership, employment, consultancies, stock ownership, or other equity interest; and expert testimony or patentlicensing arrangements), or non-financial interest (such as personal or professional relationships, affiliations, knowledge or beliefs) in the subject matter or materials discussed in this manuscript.

Funding: No funding was received for this research

\section{References}

1. Global Report on Diabetes (2016) World Health Organization.

2. Al-Nozha MM, Al-Maatouq MA, Al-Mazrou YY, Al-Harthi SS, Arafah MR, et al. (2004) Diabetes mellitus in Saudi Arabia. Saudi Med J 25: 1603-1610. 
3. StåhlmanM, Fagerberg B, Adiels M, Ekroos K, Chapman JM, et al. (2013) Dyslipidemia, but not hyperglycemia and insulin resistance is associated with marked alterations in the HDL lipidome in type 2 diabetic subjects in the DIWA cohort: Impact on small HDL particles. Biochim Biophys Acta 1831: 1609-1617.

4. Ramachandran A, Snehalatha C, Samith Shetty A, Nanditha A (2013) Primary prevention of Type 2 diabetes in South Asians--challenges and the way forward. Diabet Med 30: 26-34.

5. Klein R, Klein BE, Moss SE, DeMets DL, Kaufman I, et al. (1984) Prevalence of diabetes mellitus in southern Wisconsin. Am J Epidemiol 119: 54-61.

6. Falcão M, Falcão-Reis F, Rocha-Sousa A (2010) Diabetic retinopathy: understanding pathologic angiogenesis and exploring its treatment options. The Open Circulation and Vascular J 3: 30-42.

7. American Diabetes Association (2016) Standards of Medical care in diabetes. J Clin applied Res Education 39.

8. Wilkinson CP, Ferris FL, Klein RE, Lee PP, Agardh CD, et al. (2003) Proposed international clinical diabetic retinopathy and diabetic macular edema disease severity scales. Ophthalmology 110: 16771682.

9. Chobanian AV, Bakris GL, Black HR, Cushman WC, Green LA, et al. (2003) Seventh report of the joint national committee on prevention, detection, evaluation, and treatment of high blood pressure. Hypertension 42: 1206-1252.

10. Heart risk calculator.

11. Zhang X, Saaddine JB, Chou CF, Cotch MF, Cheng YJ, et al. (2010) Prevalence of Diabetic Retinopathy in the United States, 2005-2008. JAMA 304: 649-656.

12. Zhou Z and Ren X (2015) Consumption of Aloe vera Mucilage Attenuates Plasma Oxidative Stress and Dyslipidemia in Type 2 Diabetic Rats. Glob J Biotechnol Biomater Sci 1: 001-003.

13. Nicoli, P (2010) Modeling the intensity profiles of retinal vessels: an extended hermite model and its validation.

14. Grundy S M (2012) Pre-diabetes, metabolic syndrome, and cardiovascular risk. J Am Coll Cardiol 14; 59: 635-643.

15. Hu A, Luo Y, Li T, Guo X, Ding X, et al. (2012) Low serum apolipoprotein $A 1 / B$ ratio is associated with proliferative diabetic retinopathy in type 2 diabetes. Graefes Arch Clin Exp Ophthalmol 250: 957-962.

16. Gadi R and Samaha FF (2007) Dyslipidemia in Type 2 Diabetes Mellitus. Curr Diab Rep 7: 228-234.
17. Timothy JL, Alicia JJ, Deyi Z, Daniel TL, Daniel MW, et al. (2004) Diabetic Retinopathy and Serum Lipoprotein Subclasses in the DCCT/EDIC Cohort. Invest Ophthalmol Vis Sci 45: 3910-3918.

18. Gnaneswaran S, Vinodhini V, Kuberan, D, RajeshK, Swamy RSV, et al. (2013) Dyslipidemia and Diabetic Retinopathy in a Rural Population. Int J Res Pharmaceut Biomed Sci 4: 2229-3701.

19. Kohner EM, Aldington SJ, Stratton IM, Manley SE, Holman RR, et al. (1998) United Kingdom Prospective Diabetes Study. Diabetic retinopathy at diagnosis of non-insulin-dependent diabetes mellitus and associated risk factors. Arch Ophthalmol 116: 297-303.

20. Wong TY, Klein R, Islam FM, Cotch MF, Folsom AR, et al. (2006) Diabetic retinopathy in a multi-ethnic cohort in the United States. Am J Ophthalmol 141: 446-455.

21. Aldebasi YH, Mohieldein AH, Almansour YS , Almutairi BL (2013) Dyslipidemia and lipid peroxidation of Saudi type 2 diabetics with proliferative retinopathy. Saudi Med J 34: 616-622.

22. El-Mofty H, Abdel Hakim MA, El Din HAG, Allah OK, Mosaad, PS (2013) Retinopathy and Dyslipidemia in Type II Diabetes Mellitus in Egyptian Patients. J Clin Exp Ophthalmol 4: 265

23. Van Leiden HA, Dekker JM, Moll AC, Nijpels G, Heine RJ, et al. (2002) Blood pressure, lipids, and obesity are associated with retinopathy: the Hoorn study. Diabetes Care 25:1320-1325.

24. Klein R, Marino EK, Kuller LH, Polak JF, Tracy RP, et al. (2002) The relation of atherosclerotic cardiovascular disease to retinopathy in people with diabetes in the Cardiovascular Health Study. Br J Ophthalmol 86: 84-90.

25. Suwal B, Shrestha JK, Joshi SN, Sharma AK (2015) Diabetic retinopathy with or without clinically significant macular edema: The influencing factors. Nepal J Ophthalmol 7: 142-147.

26. Cukiernik $M$, Hileeto $D$, Evans $T$, Mukherjee $S$, Downey $D$, et al (2004) Vascular endothelial growth factor in diabetes induced early retinal abnormalities. Diabetes Res Clin Pract 65: 197-208.

27. Klein BE, Moss SE, Klein R, Surawicz TS (1991) The Wisconsin Epidemiologic Study of Diabetic Retinopathy. XIII. Relationship of serum cholesterol to retinopathy and hard exudate. Ophthalmology 98: 1261-1265.

28. Golubovic AM (2007) Association of dyslipidaemia with macular oedema and hard exudates in diabetic maculopathy. Prilozi 28: 149160.

29. Sachdev N and Sahni A (2010) Association of systemic risk factors with the severity of retinal hard exudates in a north Indian population with type 2 diabetes. J Postgrad Med 56: 3-6. 